\title{
A Scheme for Joint Signal Reconstruction in Wireless Multimedia Sensor Networks
}

\author{
Javad Afshar Jahanshahi, Habibollah Danyali, and Mohammad Sadegh \\ Helfroush \\ Department of Electrical and Electronics Engineering, Shiraz University of \\ Technology, \\ Shiraz, Iran
}

\begin{abstract}
In context aware wireless multimedia sensor networks, scenarios are usually such that signals of multiple distributed sensors contain a common sparse component and each individual signal owns an innovation sparse component. So distributed compressive sensing based on joint sparsity of a signal ensemble concept exploits both these intra- and inter- signal correlation structures and compress signals to the extent possible. This paper proposes an optimized reconstruction scheme based on joint sparsity model which is derived from the distributed compressive sensing. In this regard, based on distributed compressive sensing, a joint reconstruction scheme is proposed to compress and reconstruct ensemble of signals even in large scale data transmission. Furthermore, simulation results show the effectiveness of the proposed method in diverse compression ratios and processing times in comparison with the joint sparsity model and individual compressive sensing reconstruction methods.
\end{abstract}

Keywords: Wireless Multimedia Sensor Networks,Distributed Compressive Sensing, Joint Sparsity Model, Joint Reconstruction.

\section{Introduction}

As the field of telecommunication and new developed application advances, the need of deploying distributed Wireless Sensor Networks (WSN) and Wireless Multimedia Sensor Networks (WMSNs) which consist of many sensors for monitoring a specific phenomenon in an area of interest both in time and space is increased. There are three main challenges in WSN, i.e., network lifetime, computational ability and bandwidth constraints $[1,2]$. Assume that there are $J$ sensors in the area which are measuring a phenomenon in the spatio-temporal manner. A fusion center (FC) receives all the measurements shown in (Fig. 1) and runs an algorithm to jointly decode signal ensembles of the sensors and reconstruct the phenomenon at the sensor sides. In such cases, when there is a significant correlation between the signals of the sensors, the joint decoding based on the distributed source coding (DSC) [3] could be used in the FC to decompress the transmitted signals of the sensors. Using DSC methods, the compression rate can be higher and thus, data can be transmitted with less power and bandwidth consumption. DSC concepts are

DOI: 10.5121/ijma.2017.9611. 
also used to improve the wireless sensor networks' performance in terms of data reception accuracy and energy consumption efficiency [4].

In addition, compressive sensing (CS) provides a simultaneous sensing and compression framework [5], enabling a potentially significant reduction in the sampling and computation costs at a sensor with limited capabilities. The CS theory shows that a signal with a sparse transform representation can be reconstructed from a small set of incoherent random projections. CS scheme is exploited in many research fields such as radar imaging [6], cognitive spectrum sensing [7,8], channel estimating [9] , error corrections [11], antenna array synthesis problems [10] and so on. The CS technique as the data acquisition approach in a WMSN can significantly reduce the energy consumed in the process of sampling and transmission through the network, and also lower the wireless bandwidth required for communication.

In wireless sensor networks, sensors have limited computation capability and energy resources without assistance of any established infrastructures, so many studies in the literature are conducted considering these limitations for various applications [13]. Recently, the theory of distributed compressive sensing (DCS) [12] have been used to exploit inter- and intra-signal correlations to propose new distributed source coding and compression algorithms for multi-signal ensembles based on the CS theory. In a typical DCS setting and joint sparsity model (JSM) [12], each sensor compresses its signal independently by projecting it onto an incoherent basis and transmits the compressed information (actually sensed) to the FC. Under the right conditions the $\mathrm{FC}$ can jointly reconstruct all the signals by knowing that the measured signals of each sensor are individually sparse in some basis.

This present paper goes in the direction of proposing a framework for compressing the signals of a WMSN and reducing the amount of the transmitted data as much as possible. In the WMSN, the captured signals of each sensor should be transmitted (direct or with a multi-hop architecture) to a central unit (FC) through a wireless interface. In this paper, we address the problem of joint reconstruction ensemble of signals for sensor nodes. The proposed model denoted as Enhanced Common Method (ECM) basically can be used in WMSN's services where an intrinsic shared part exists between the signals of the nodes that can be represented sparsely in a dictionary or basis. It means that ECM is a context aware algorithm and depends on the properties of the captured signals. Another aim of the ECM framework is ability to robustly reconstruct the sensors' signals if some disturbances occur in the transmission procedure.

The rest of this paper is organized as follow; the system model and our approach is described in Section 2 and 3, respectively. The corresponding experimental results are presented and discussed in Section 4. Finally, the paper is concluded in Section 5. 


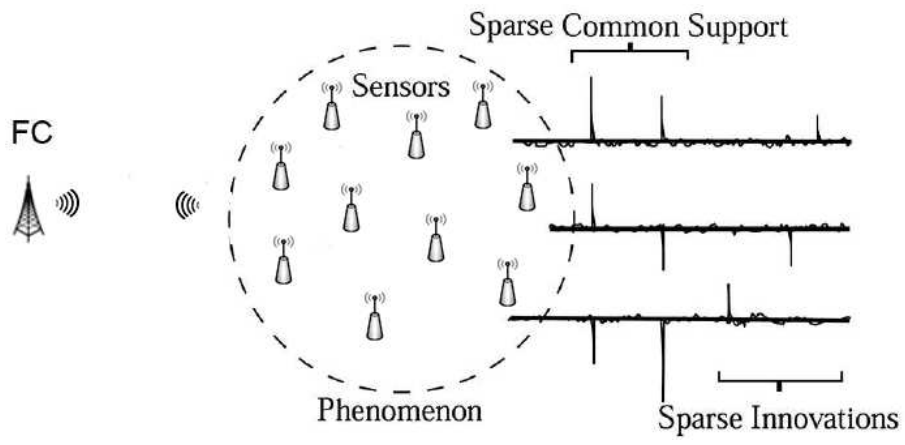

Fig. 1. A collection of sensors measure a phenomenon and send their measurements to the fusion center. There exists some inter-relation between the signals and FC decodes them jointly.

\section{System Model}

Suppose that $J$ sensors are distributed in the area and each of them capture a signal $\boldsymbol{x}_{j} \in R^{N}(j \in 1,2, \ldots, J)$. All $J$ signals share a common component $\boldsymbol{x}_{c} \in R^{N}$ such that $\boldsymbol{x}_{j}=\boldsymbol{x}_{c}+\boldsymbol{x}_{i n n_{j}}$ where $\boldsymbol{x}_{i n n_{j}} \in R^{N}$ is the innovation part of each signal $\boldsymbol{x}_{j}$. There is a dictionary $D \in R^{N \times K}$ in which signals can be represented sparsely $\left(\boldsymbol{x}_{j}=D \boldsymbol{\alpha}_{j}\right)$ as a linear combination of the atoms (columns) of this dictionary. Obviously, this dictionary has the ability to represent signals sparsely as Eq. (1) which $\boldsymbol{\alpha}_{c}$ and $\boldsymbol{\alpha}_{i n n_{j}}$ s are belonged to space $R^{K}$ with different sparsity levels.

$$
\begin{gathered}
\boldsymbol{x}_{j}=D \boldsymbol{\alpha}_{j}=D\left(\boldsymbol{\alpha}_{c}+\boldsymbol{\alpha}_{i n n_{j}}\right), \\
\boldsymbol{x}_{c}=D \boldsymbol{\alpha}_{c},\left\|\boldsymbol{\alpha}_{c}\right\|_{0}=k_{c} \\
\boldsymbol{x}_{i n n_{j}}=D \boldsymbol{\alpha}_{i n n_{j}},\left\|\boldsymbol{\alpha}_{i n n_{j}}\right\|_{0}=k_{j}
\end{gathered}
$$

Each sensor compressed (actually sensed) its signal $\boldsymbol{y}_{j}=\Phi_{j} \boldsymbol{x}_{j}$ by using an individual measurement matrix $\Phi_{j} \in R^{w_{j} \times N}$. The samples of the sensed signal $\boldsymbol{y}_{j} \in R^{w_{j}}$ of each sensor should be sent to and consequently detected in the fusion center via a conventional digital communication transceiver module. Obviously, $\boldsymbol{y}_{j}$ is the combination of two parts: one is the common part $\boldsymbol{y}_{c_{j}} \in R^{w_{j}}$ and another is the innovation part $\boldsymbol{y}_{i n n_{j}} \in R^{w_{j}}$, which can be expressed as:

$$
\begin{gathered}
\boldsymbol{y}_{c_{j}}=\Phi_{j} \boldsymbol{x}_{c}, \\
\boldsymbol{y}_{i n n_{j}}=\Phi_{j} \boldsymbol{x}_{i n n_{j}},
\end{gathered}
$$

Therefore, it is possible to write that

$$
\boldsymbol{r}_{j}=\boldsymbol{r}_{c_{j}}+\boldsymbol{r}_{i n n_{j}}
$$


where $\boldsymbol{r}_{c_{j}}$ and $\boldsymbol{r}_{i n n_{j}}$ are the received signals in the FC corresponding to the common and innovation parts of the transmitted one.

Inspired from JSM models, we define the Common Method to recover and decompress the data as follows:

$$
\begin{aligned}
& \boldsymbol{r}=\Phi \Psi \boldsymbol{\alpha}+\boldsymbol{n} \\
& \boldsymbol{r}=\left[\begin{array}{c}
\boldsymbol{r}_{1} \\
\boldsymbol{r}_{2} \\
\vdots \\
\boldsymbol{r}_{J}
\end{array}\right], \boldsymbol{n}=\left[\begin{array}{c}
\boldsymbol{n}_{1} \\
\boldsymbol{n}_{2} \\
\vdots \\
\boldsymbol{n}_{J}
\end{array}\right], \boldsymbol{\alpha}=\left[\begin{array}{c}
\boldsymbol{\alpha}_{c} \\
\boldsymbol{\alpha}_{i n n_{1}} \\
\vdots \\
\boldsymbol{\alpha}_{i n_{J}}
\end{array}\right] \\
& \Phi=\left[\begin{array}{cccc}
\Phi_{1} & 0 & \cdots & 0 \\
0 & \Phi_{2} & \cdots & 0 \\
\vdots & & \ddots & \vdots \\
0 & \cdots & 0 & \Phi_{J}
\end{array}\right], \Psi=\left[\begin{array}{ccccc}
D & D & 0 & \cdots & 0 \\
D & 0 & D & \cdots & \vdots \\
\vdots & & \ddots & 0 \\
D & 0 & \cdots & 0 & D
\end{array}\right]
\end{aligned}
$$

where $\boldsymbol{r} \in R^{W}, \boldsymbol{n} \in R^{W}, \boldsymbol{\alpha} \in R^{K(J+1)}, \boldsymbol{\Phi} \in R^{W \times N J}, \boldsymbol{\Psi} \in R^{J N \times K(J+1)}$ and $W=\sum_{j=1}^{J} w_{j}$. In order to recover the desired signals, first $\hat{\boldsymbol{\alpha}}=\left[\begin{array}{lll}\hat{\boldsymbol{\alpha}}_{c}^{T} & \hat{\boldsymbol{\alpha}}_{i n n_{1}}^{T} \cdots \hat{\boldsymbol{\alpha}}_{i n_{J}}^{T}\end{array}\right]^{T}$ is computed by solving the optimization problem in Eq. (7).

$$
\hat{\boldsymbol{\alpha}}=\min _{\dot{\alpha}}\|\dot{\boldsymbol{\alpha}}\|_{0 \text { or } 1} \text { subject to }\|\boldsymbol{r}-\Phi \Psi \dot{\boldsymbol{\alpha}}\|_{2} \leq \epsilon
$$

Then $\hat{\boldsymbol{x}}_{j}$ will be computed by

$$
\hat{\boldsymbol{x}}_{j}=D\left(\hat{\boldsymbol{\alpha}}_{c}+\hat{\boldsymbol{\alpha}}_{i n n_{j}}\right)
$$

where $\hat{\boldsymbol{\alpha}}_{c}$ and $\hat{\boldsymbol{\alpha}}_{i n n_{j}}$ s are located in the found $\hat{\boldsymbol{\alpha}}$ vector.

\section{The Proposed Enhanced Common Method}

In order to recover the uncompressed signals $\boldsymbol{x}_{j}$ more robustly, an enhanced common model $(E C M)$ is proposed which uses the shared common component $\boldsymbol{x}_{c}$ based on the JSM model [12] concepts and also can recover the data in the lower measuring rate. However, firstly, we assume the common part $\boldsymbol{\alpha}_{c}$ to be known by the fusion center. This phenomenon can be used in the receiver (FC) to enhance the reconstruction performance and system's efficiency. Hence, in order to enhance the 
reconstruction performance, we remove the common part from the reconstruction Eq. (7) as follows:

$$
\boldsymbol{r}=[\Phi \Psi]\left[\begin{array}{c}
\boldsymbol{\alpha}_{c} \\
\boldsymbol{\alpha}_{i n n_{1}} \\
\vdots \\
\boldsymbol{\alpha}_{i n n_{J}}
\end{array}\right]+\boldsymbol{n}
$$

and it can be rewritten to Eq. (10) by using a combination of two distinct parts:

- Common part $\boldsymbol{\alpha}_{c}$

- Innovation part $\boldsymbol{\alpha}_{I}=\left[\begin{array}{llll}\boldsymbol{\alpha}_{i n n_{1}}{ }^{T} & \boldsymbol{\alpha}_{i n n_{2}}{ }^{T} & \cdots & \boldsymbol{\alpha}_{i n n_{J}}{ }^{T}\end{array}\right]^{T}$

$$
\boldsymbol{r}=[A \| H]\left[\begin{array}{c}
\boldsymbol{\alpha}_{c} \\
-- \\
\boldsymbol{\alpha}_{i n n_{1}} \\
\vdots \\
\boldsymbol{\alpha}_{i n_{J}}
\end{array}\right]+\boldsymbol{n}=A \boldsymbol{\alpha}_{c}+H \boldsymbol{\alpha}_{I}+\boldsymbol{n}
$$

If we define $\boldsymbol{r}_{i n n}=\boldsymbol{r}-A \boldsymbol{\alpha}_{c}$, the reconstruction formula is modified to find just the innovation parts of the signals:

$$
\hat{\boldsymbol{\alpha}}_{I}=\min _{\boldsymbol{\alpha}_{I}^{\prime}}\left\|\boldsymbol{\alpha}_{I}^{\prime}\right\|_{0 \text { or } 1} \text { subject to }\left\|\boldsymbol{r}_{\text {inn }}-H \boldsymbol{\alpha}_{I}^{\prime}\right\|_{2} \leq \epsilon
$$

Consequently, the signal of each sensor $\hat{\boldsymbol{x}}_{j}$ is found by

$$
\hat{\boldsymbol{x}}_{j}=D\left(\boldsymbol{\alpha}_{c}+\hat{\boldsymbol{\alpha}}_{i n n_{j}}\right)
$$

where $\hat{\boldsymbol{\alpha}}_{i n n_{j}}$ s are located in the computed $\hat{\boldsymbol{\alpha}}_{I}$ vector. This modification is indicated to bring faster solution and also better reconstruction accuracy. Moreover, in order to improve the reconstruction accuracy and solution time of the recovery algorithm, we removed the common components from the recovery algorithm.

\section{Experimental Results}

In order to show the ability of the proposed models some experimental results are reported here. In the following experiments, five signals $\boldsymbol{x}_{j} \in R^{400}, j=\{1,2, \ldots, 5\}$ from $J=5$ sensors are generated such that there is a shared common component $\boldsymbol{x}_{c} \in R^{400}$ between them and also each of them are sparse in a random dictionary $D \in R^{400 \times 512}$ with different sparsity levels (maximum 50-sparse). Consequently, the signals are sensed by five different measurement matrices $\Phi_{j} \in R^{w_{j} \times 400}$ with 
Gaussian random set of projections. The sensed samples $\boldsymbol{y}_{j} \in R^{w_{j}}, j=\{1,2, \ldots, 5\}$ are then sent to the fusion center through a digital transceiver system. Binary phase shift keying modulating (BPSK), 1/2 channel encoding, and DS-CDMA with 4chip's length are the specifications of the used transceiver system. Simulations are experimented for 100 frames with different $\boldsymbol{x}_{j}$ s and the obtained mean results are reported.

The CVX Matlab toolbox $[16,17]$ is used to solve the least square problem in Eq. (11). The CVX is a programming package for specifying and solving convex programs. Moreover, other sparse-based optimization problems in this paper are solved using basis pursuit denoising (BPDN) relaxation method [14] which is more robust than other conventional algorithms against the incomplete and inaccurate samples. We also use the SparseLab [15] Matlab toolbox to run the BPDN algorithm. SparseLab is a Matlab software package designed to find sparse solutions to systems of linear equations, particularly under-determined systems.

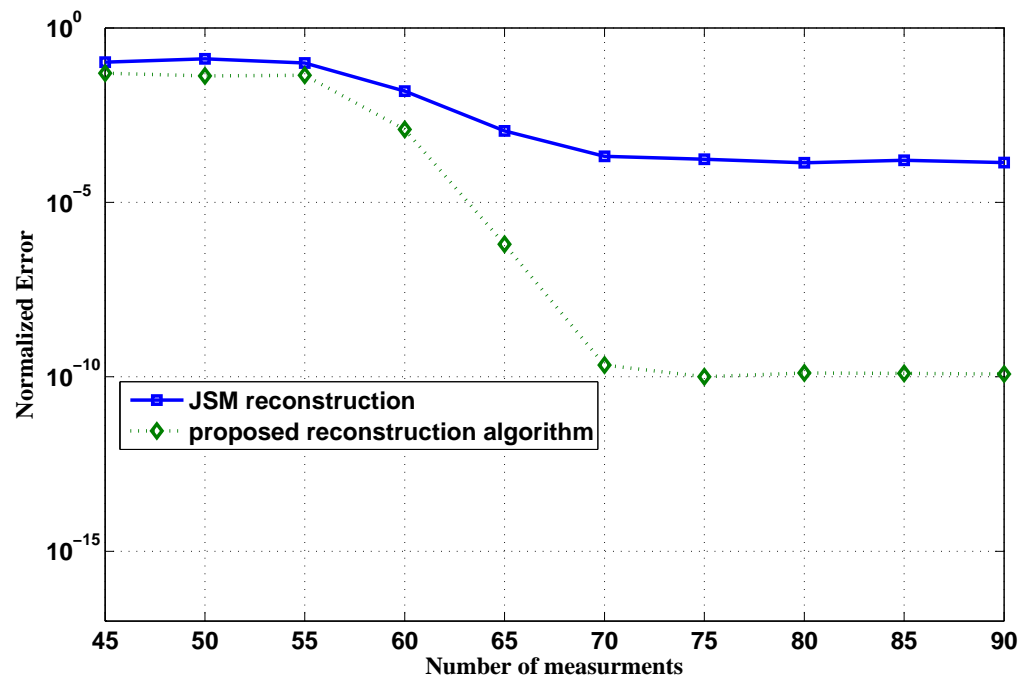

Fig. 2. Comparison between compression ability of the proposed models in a loss-less scenario.

Fig. 2 shows the reconstruction performance of all the proposed methods for different sensing $\left(w_{j}\right)$ and compression rates evaluated by semi normalized mean squared error (NMSE). The sensing rate and the compression rate can be computed as $\frac{w_{j}}{400}$ and $1-\frac{w_{j}}{400}$, respectively. The normalized mean squared error (NMSE) which 
we define for our experiments is as follows:

$$
N M S E=\frac{1}{J N} \sum_{j=1}^{J} \sum_{n=1}^{N}\left(\frac{\widehat{\boldsymbol{x}}_{j}(n)}{\left\|\widehat{\boldsymbol{x}}_{j}\right\|_{2}}-\frac{\boldsymbol{x}_{j}(n)}{\left\|\boldsymbol{x}_{j}\right\|_{2}}\right)^{2}
$$

where $J$ and $N$ are the number of sensors and signals' samples, respectively. Note that, the defined semi normalized mean squared error calculates the mean (with respect to the number of sensors) of the sum of all differences between the actual and reconstructed samples of each sensor's signal. It means that, the averaging process is only performed with respect to $J$ and $N$ which can cause the computed semi normalized mean squared error to be larger than 1 . It can be inferred from Fig. 2 that using the Enhanced Common Model, as compared to the JSM model, causes signals to be reconstructed more efficiently and in a lower sensing rate. This fact is based on that, removing the known common part from the reconstruction formula and reducing the length of the desired sparse vector makes the finding of the sparse vector to be more accurate. Moreover, Fig. 2 concludes and shows the accuracy of signal reconstruction for different sensing rates by using the proposed method and the state of the art JSM model. However, the performance shown in Fig. 2 is achieved when the sensed samples $\boldsymbol{y}_{j} \in R^{w_{j}}, j=\{1,2, \ldots, 5\}$ are sent to the fusion center through a lossless system without any symbol errors. In fact, Fig. 2 was just reported to show the compression ability of the proposed methods.

In order to find a sense about the reconstruction performance of the semi normalized mean squared error and sparsity property of the $\boldsymbol{\alpha}_{j} \mathrm{~s}$, Figures 3 and 4 are presented. Fig. 3 illustrates a frame of the signal of a sensor $\boldsymbol{x}_{j}$ and its reconstructed version $\hat{\boldsymbol{x}}_{j}$ by using the ECM algorithm and JSM reconstruction method. The reconstruction accuracy is compared between the two methods in Fig. 3. Moreover, corresponding to the signal shown in the Fig. 3 the original related sparse vectors $\boldsymbol{\alpha}_{j}$ s and their computed versions $\hat{\boldsymbol{\alpha}}_{j}$ s by FC are shown in Fig. 4.

\section{Conclusion}

In this paper, an enhanced common framework is proposed to compress the captured signals of the sensors which should be transmitted to FC. the proposed ECM basically can be used in WMSN services where an intrinsic shared part exists between the sensors' captured signals and also the signals can be sparsely represented in a dictionary or basis. This makes ECM to be a context aware method and useful in many applications. Because of compressing the signals of the sensors and therefore, lower transmitted data, ECM can be used in lower bandwidth usage. Furthermore, since compressive sensing sampling method is used to compress the signals of the sensors, using ECM brings lower computational cost and therefore, greater lifetime in the sensor side. 


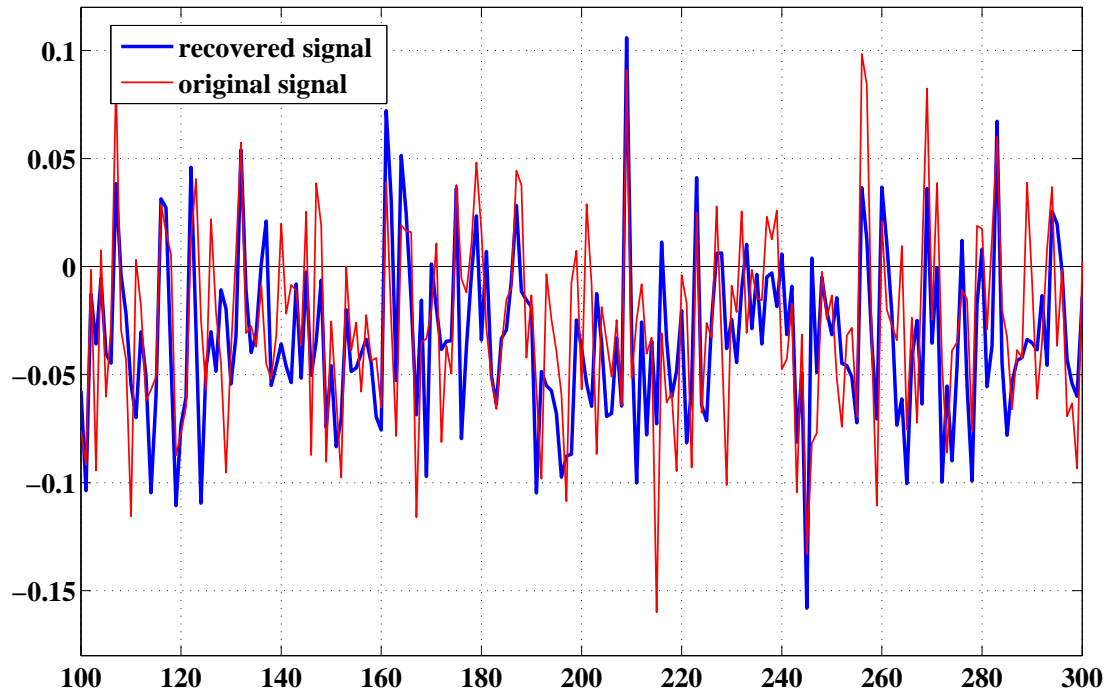

(a) Reconstructed with JSM reconstruction method

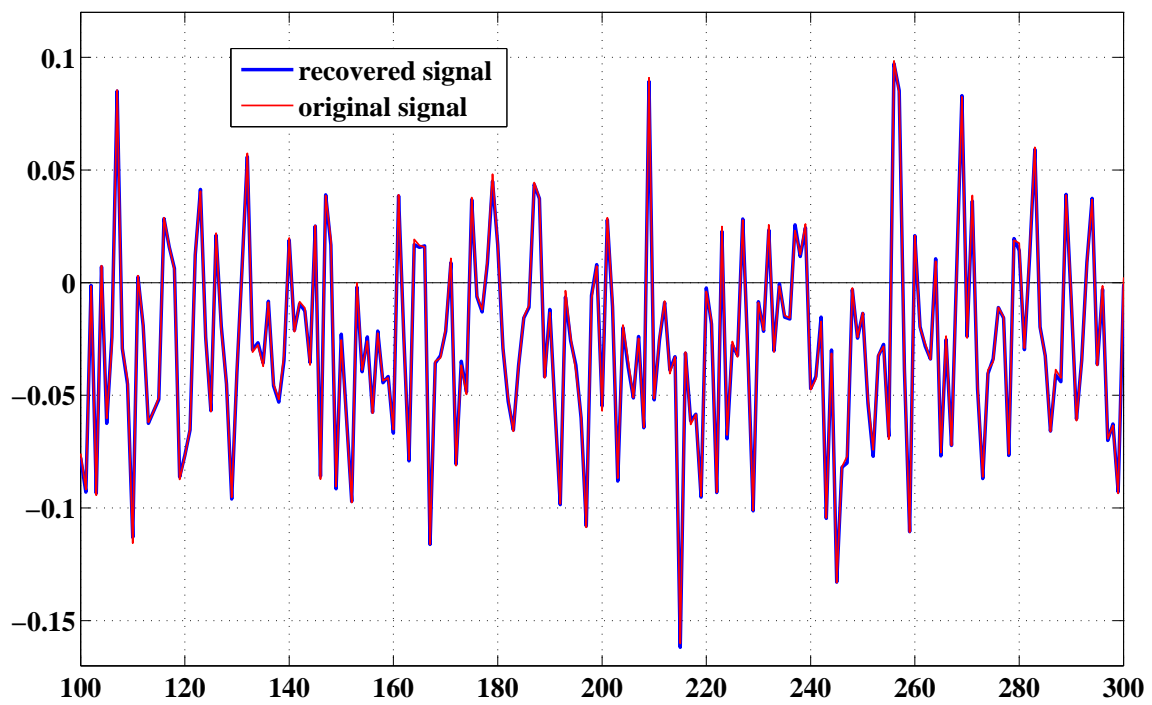

(b) Reconstructed with ECM algorithm

Fig. 3. A frame of the signal of a sensor $\boldsymbol{x}_{j}$ and its reconstructed versions $\hat{\boldsymbol{x}}_{j}$ by FC. 
The International Journal of Multimedia \& Its Applications (IJMA) Vol.9, No.4/5/6, December 2017



(a) Reconstructed with JSM reconstruction method

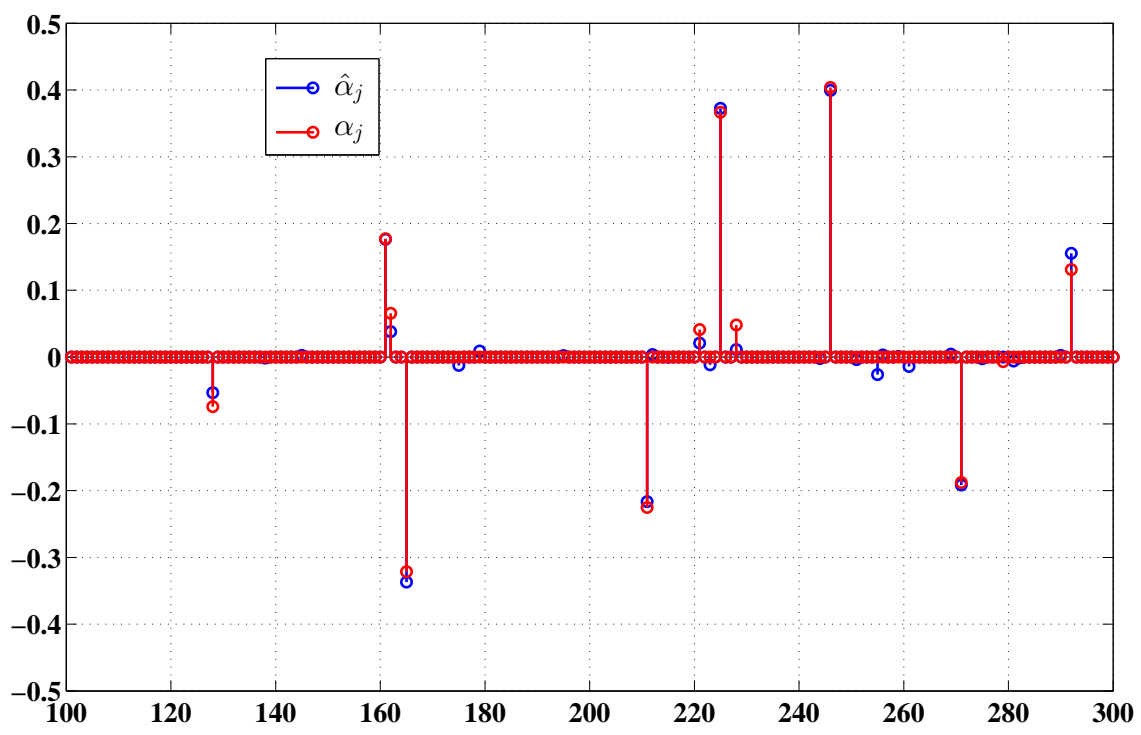

(b) Reconstructed with ECM algorithm

Fig. 4. Sparse vectors $\boldsymbol{\alpha}_{j}$ and their reconstructed versions $\hat{\boldsymbol{\alpha}}_{j}$ corresponding to the signal of the Fig. 3. 
The International Journal of Multimedia \& Its Applications (IJMA) Vol.9, No.4/5/6, December 2017

\section{References}

1. Akyildiz, I.F. (2008). Wireless Multimedia Sensor Networks: Applications and Testbeds. Proceedings of the IEEE, vol. 96 no. 10, p. 1588 - 1605.

2. Adetoba, B.T., Awodele, O. and Kuyoro, S.O., (2017). A Multimedia Data Mining Framework for Monitoring E-Examination Environment. The International Journal of Multimedia \& Its Applications (IJMA), vol. 9, no. 3, p. 25-34.

3. Wang, W., Peng, D., Wang, H., Chen, H. (2011). Cross-layer Multirate Interaction with Distributed Source Coding in Wireless Sensor Networks. IEEE Transactions on Wireless Communications, vol. 8 no. 2, p. 787-795.

4. Hua, G., Chen, C.W. (2008). Correlated data gathering in wireless sensor networks based on distributed source coding. International Journal of Sensor Networks (IJSNET), vol. 4 no.1/2, p.13- 22 .

5. Donoho, D.L. (2006). Compressed sensing. IEEE Transaction on Information Theory, vol. 52, no. 4, p. $1289-1306$.

6. Potter, L.C., Ertin, E., Parker, J.T., Cetin, M. (2010). Sparsity and Compressed Sensing in Radar Imaging. Proceedings of the IEEE, vol. 98, no.6, p. 1006 - 1020.

7. Jahanshahi, J.A., Eslami, M., Ghorashi, S.A. (2012). PSD Map Construction Scheme Based on Compressive Sensing in Cognitive Radio Networks. IEICE Transaction on Communications, vol. 95, no.4, p. 1056-1065.

8. Jahanshahi, J.A., Eslami, M., Ghorashi, S.A. (2013). Compressed Sensing Based Dynamic PSD Map Construction in Cognitive Radio Networks, RADIOENGINEERING, vol. 22, no. 2, p. 526-535.

9. Haupt, J., Bajwa, W.U., Raz, G., Nowak, R. (2010). Toeplitz Compressed Sensing Matrices With Applications to Sparse Channel Estimation. Proceedings of the IEEE, vol. 56, no. 11, p. $5862-5875$.

10. Chen, H., WAN, Q., FAN, R. (2013). Beam Pattern Synthesis Using Reweighted L1-Norm Minimization and Array Orientation Diversity, RADIOENGINEERING, vol. 22, no. 2, p. 602-609.

11. He, Zaixing, Ogawa, T., Haseyama, M., Zhao, X., Zhang, S. (2013). A Compressed SensingBased Low-Density Parity-Check Real-Number Code, RADIOENGINEERING, vol. 22, no. 3, p.851-860.

12. Baron, D., Wakin, M.B., Duarte, M.F., Sarvotham, S., Baraniuk, R. (2006). Distributed Compressed Sensing. Preprint, arXiv:0901.3403v1, http://arxiv.org/pdf/0901.3403v1.pdf.

13. Alzoubaidi, A.R., Al-Sous, T. and Al-Bahadili, H. (2016). Adaptive-Quality Image Compression Algorithm. The International Journal of Multimedia \& Its Applications (IJMA), vol. 8, no. $3 / 4$, p. $15-26$.

14. Gill, P.R., Wang, A., Molnar, A. (2011). The In-Crowd Algorithm for Fast Basis Pursuit Denoising. IEEE Transactions on Signal Processing, vol. 59, no.10, p. 4595 - 4605.

15. Sparselab, http://sparselab.stanford.edu/.

16. CVX toolbox, http://cvxr.com/cvx.

17. Grant, M., Boyd. S. (2008). Graph implementations for nonsmooth convex programs, Recent Advances in Learning and Control (a tribute to M. Vidyasagar), V. Blondel, S. Boyd, and H. Kimura, Lecture Notes in Control and Information Sciences, p. 95-110. 


\section{Authors}

Javad Afshar Jahanshahi received his B.Sc. and M.Sc. Degrees both in Electrical Engineering from the University of the Sistan Baluchestan and Shahid Beheshti University, in 2006 and 2010, respectively. Now, he is pursuing Ph.D. program at the Department of Telecommunication Engineering, Shiraz University of Technology. His main research interests are in wireless communications and image processing.

Habibollah Danyali received the B.Sc. and M.Sc. degrees in Electrical Engineering respectively from the Isfahan University of Technology, Isfahan, Iran, in 1991 and the Tarbiat Modarres University, Tehran, Iran, in 1993. From 1994 to 2000 he was with the Department of Electrical Engineering, University of Kurdistan, Sanandaj, Iran, as a lecturer. In 2004 he received his Ph.D. degree in Computer Engineering from the University of Wollongong, Australia. After finishing his $\mathrm{PhD}$ he continued his academic work with University of Kurdistan as an assistant professor. As of September 2009, he is with the Department of Telecommunication Engineering, Shiraz University of Technology, Shiraz, Iran. His research interests include data hiding, scalable image and video coding, medical image processing and biometrics.

Mohammad Sadegh Helfroush received the B.Sc. and M.Sc. degrees in Electrical engineering from Shiraz University of Shiraz and Sharif University of Technology, Tehran in 1993 and 1995, respectively. He performed his Ph.D. degree in Electrical Engineering from Tarbiat Modarres University, Tehran, Iran. Actually he is working as associate professor in department of Electrical and Electronics Engineering, Shiraz University of Technology, Shiraz, Iran. 